\title{
One testing method of dynamic linearity of an accelerometer
}

\author{
Jing-Yu Lei ${ }^{1}$, Wei-Guo Guo ${ }^{1}$, Xue-Ming Tan ${ }^{1}$, and Yun-Bo Shi ${ }^{2}$ \\ ${ }^{1}$ School of Aeronautics, Northwestern Polytechnical University, Xi'an, Shaanxi 710072, China \\ ${ }^{2}$ School of Instrument and Electronics, North University of China, Taiyuan 030051, China
}

\begin{abstract}
To effectively test dynamic linearity of an accelerometer over a wide rang of $10^{4} \mathrm{~g}$ to about $20 \times 10^{4} \mathrm{~g}$, one published patent technology is first experimentally verified and analysed, and its deficient is presented, then based on stress wave propagation theory on the thin long bar, the relation between the strain signal and the corresponding acceleration signal is obtained, one special link of two coaxial projectile is developed. These two coaxial metal cylinders (inner cylinder and circular tube) are used as projectiles, to prevent their mutual slip inside the gun barrel during movement, the one end of two projectiles is always fastened by small screws. Ti6-AL4-V bar with diameter of $30 \mathrm{~mm}$ is used to propagate loading stress pulse. The resultant compression wave can be measured by the strain gauges on the bar, and a half -sine strain pulse is obtained. The measuring accelerometer is attached on the other end of the bar by a vacuum clamp. In this clamp, the accelerometer only bear compression wave, the reflected tension pulse make the accelerometer off the bar. Using this system, dynamic linearity measurement of accelerometer can be easily tested in wider range of acceleration values. And a really measuring results are presented.
\end{abstract}

\section{Introduction}

In the early 80s of last century, Robert D [1] in ENDEVCO corporation, Togami [2] and Ueda [3-5] had introduced the operation system for accelerometer calibration in the range of $10,000 \mathrm{~g}$ to greater than $100,000 \mathrm{~g}$. This system works based on a Hopkinson bar, and calibration of an accelerometer is based on a strain gauge measurement of the wave. Li et al. [6] also presents the modified Hopkinson bar system, this system can calibrate the accelerometer in a range from 5,000 g to $200,000 \mathrm{~g}$. In this system, a half-sine acceleration pulse is obtained by impacting a parabolic pointed projectile. To achieve a wider range acceleration calibration, different shapes of projectiles have to be used.

According to the measuring method of dynamic linearity for the accelerometer presented by references $[7,8]$, in dynamic linearity measurement, except each projectile is alone fired, two projectiles should impinge against the end surface of the Hopkinson bar at the same time as when it is fired alone. In reality, it is hard to achieve such perfect results because two projectiles synchronization is not easy obtained by using independent double gun barrels.

In this paper, a more easy measuring method has been proposed, that is, we base on such a fact, the acceleration pulse is always located in first half of a loading strain pulse in the Hopkinson shock test [1-6], and combining with the relation between projectile shape and strain wave, two coaxial projectiles (outer circular tube and inner cylinder) are always fired by a single gun barrel during whole operation. This replace the coaxial double gun barrel, and the synchronization impact on the end surface of the bar is effectively ensured, so it is much more convenient for dynamic linearity measurement.

\section{Measurement of accelerometer dynamic linearity}

Reference [7] and [8] have introduced one disclosed method of dynamic linearity measurement, and a schematic diagram of measuring test is presented as Fig. 1. In the figure, a coaxial double gun barrel must be used, when an outer projectile (circular tube) impinges against the end surface of incident bar, the impact acceleration pulse $a_{1}(t)$ of the accelerometer is generated. In the same way, $a_{2}(t)$ is obtained when an inner projectile impacts to the bar. And then, when these two projectiles are lunched together to impinge against the surface of incident bar at the same time as when it is fired alone, third superimposition acceleration can be obtained, that is $a_{1+2}(t)$. And the following expression is held as long as the strain of the pulse waves keep the linear elastic deformation of the bar material.

$$
\begin{aligned}
& \varepsilon_{1+2}(t)=\alpha \varepsilon_{1}(t)+\beta \varepsilon_{2}(t) \\
& a_{1+2}(t)=\alpha a_{1}(t)+\beta a_{2}(t)
\end{aligned}
$$

where $\alpha$ and $\beta$ are arbitrary constants, formulas (1) and (2) are foundation of calibration of accelerometer dynamic linearity.

In Fig. 1, the experimental setup takes a double gun barrels (inner and outer tube) to fire coaxial projectiles. When inner projectile is first fired, the output signal from an accelerometer is $g_{1}(t)$ (here, $g_{1}(t)=a_{1}(t) / 10$ ), then outer projectile is $g_{2}(t)$; finally inner and outer projectile are simultaneously fired, output pulse is $g_{1+2}(t)$. Based on the definition of accelerometer dynamic linearity, the 


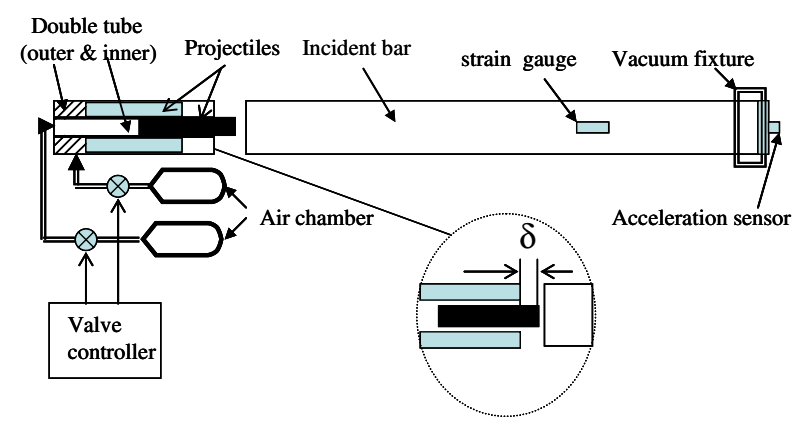

Figure 1. Accelerometer dynamic linearity by double gun barrels.

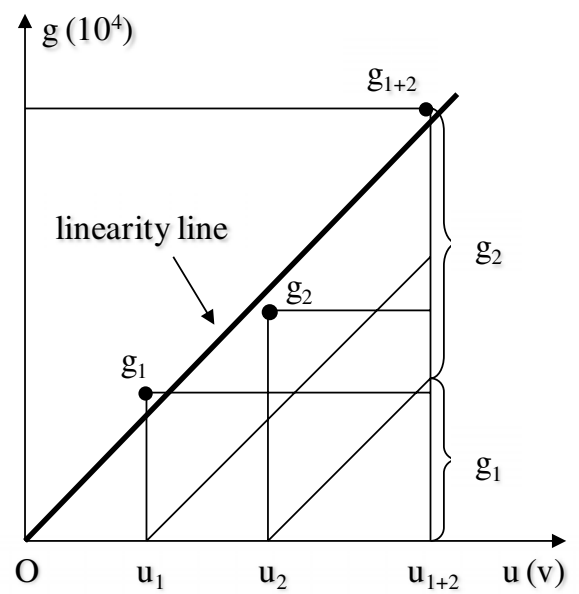

Figure 2. Diagrammatic sketch of dynamic linearity of accelerometer.

following formula can be established

$$
g_{1+2}(t)=\alpha g_{1}(t)+\beta g_{2}(t) .
$$

Through computation, we can evaluate dynamic linearity of accelerometer. In testing procedure, $g_{1}(t)$ and $g_{2}(t)$ are obtained by independently firing oneself projectile (the inner or outer one alone); $g_{1+2}(t)$ is obtained by simultaneously firing inner and outer projectiles. If the max acceleration value only is used to evaluate an accelerometer, the dynamic linearity of accelerometer is obtained in Fig. 2.

The principal problem of this measuring method is, the synchronous impacting contact of two independent projectiles on the end of incident bar must be achieved in third testing step.

If there is a little synchronous difference, it is limited that this synchronous time difference is not allowed over $10 \mu \mathrm{s}[7,8]$, but in practice we usually obtain the maximum displacement difference of the two strain pulse as shown in the Fig. 3. The $\delta_{1}$ is usually more over $10 \mu \mathrm{s}$, which does not meet the requirement.

It is abstractly known that, in order to obtain $a_{1+2}(t)$, the outer and inner projectiles impact against the end surface of incident bar at the same time just as they are fired alone. In real tests, two projectiles are usually fired through valve controller, as shown in Fig. 1, and so through the independent air tubes, the gun barrels and air chambers etc., these will affect the moving synchronization of these

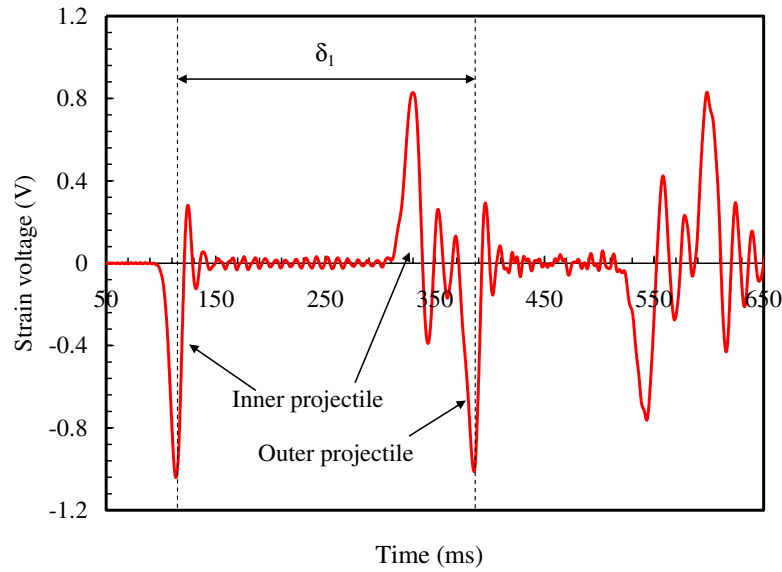

Figure 3. Strain pulse time difference under synchronous projectile firing.

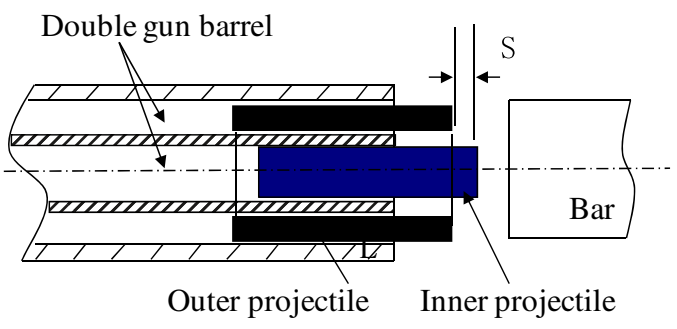

Figure 4. The schematic diagram of the displacement difference of two projectiles.

\begin{tabular}{|l|l|}
\hline $\mathrm{V}(\mathrm{m} / \mathrm{s})$ & $\triangle \mathrm{S}(\mathrm{mm})$ \\
\hline 1 & 0.01 \\
\hline 5 & 0.05 \\
\hline 10 & 0.1 \\
\hline 15 & 0.15 \\
\hline 20 & 0.2 \\
\hline 25 & 0.25 \\
\hline 30 & 0.3 \\
\hline 35 & 0.35 \\
\hline
\end{tabular}

Figure 5. Max allowable displacement difference for two projectile impact.

two projectiles, and make it hard to synchronously impinge the end surface of incident bar under these uncertain factors.

Figures 4 and 5 further present a max allowable displacement difference if two projectiles synchronously impact in an allowable $10 \mu$ s time difference, e.g. a max 


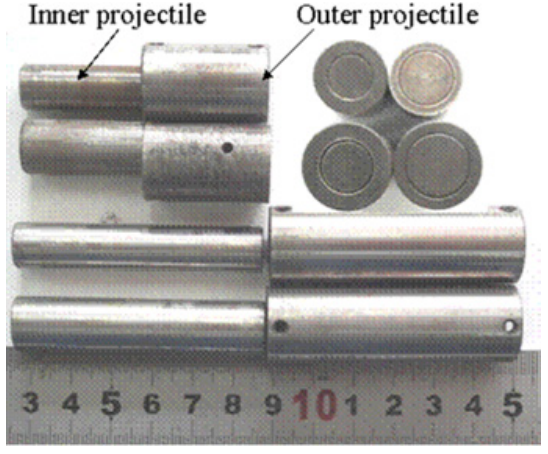

Figure 6. Pictures of the coaxial cylinder projectiles with different diameters and lengths.

allowable displacement difference is not over $0.1 \mathrm{~mm}$ $(\Delta S=0.1 \mathrm{~mm})$ if the speed of two projectiles is about $10 \mathrm{~m} / \mathrm{s}$.

\section{Modified measuring method}

As known in the references 1 to 6 , a wider range of acceleration values can be achieved by the single gun barrel device. However, in practice, the entire range of acceleration value up to about $200,000 \mathrm{~g}$ cannot be achieved by only one type projectile. Such two inevitable point are: (1) two types of projectiles have to be applied; (2) different types of projectiles can results in different acceleration pulse width, frequency components, etc.

Meanwhile, it is aware that, the acceleration pulse of the accelerometer is always located in the first half strain pulse on the bar. Based on this, we still take single barrel gun, but two projectiles (inner and outer projectiles) are simultaneously fired at any time, two coaxial cylinder projectiles in single barrel are illustrated in Fig. 6, and their contact faces are greased to reduce reaction friction force, while different length of projectiles can be used to obtain different wide acceleration pulse.

\section{Real measuring demonstration}

In this test, Ti-6Al-4V titanium rod was used as stress pulse propagating bar, it is $1200 \mathrm{~mm}$ long with $30 \mathrm{~mm}$ diameter. The projectiles are made into coaxial cylinders, the inner cylinder (inner projectile) and the circular tube (outer projectile, here its max diameter is $20 \mathrm{~mm}$ ) can be the same cross section area, they can also be made into different section area. Two projectiles can be manufactured with different lengths, the length and cross section of projectiles depends on required $g$ value and frequency width. Two sets real projectiles are shown in Fig. 6.

See Fig. 4, the overrun gap $S$ of two coaxial cylinders projectiles is always set to about $4 \mathrm{~mm}$, their rear-end is always fastened by small screws to prevent the relative slip of two projectiles when they moved inside the barrel. Whole experimental procedure is divided into three steps, in step 1 , the inner projectile is fired at a fixed velocity of $10 \mathrm{~m} / \mathrm{s}$, the strain history $\varepsilon_{1}(t)$ on the bar and the accelerometer output pulse $g_{1}(t)$ are obtained; and then in step 2 , the circular tube is fired at a same velocity of $10 \mathrm{~m} / \mathrm{s}, \varepsilon_{2}(t)$ and $g_{2}(t)$ are obtained, finally in step 3,

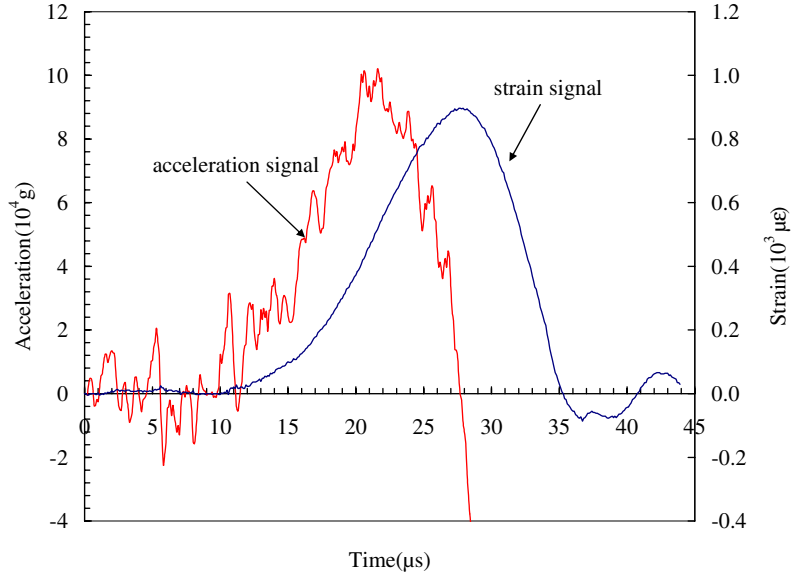

Figure 7. Acceleration and corresponding strain pulses on the bar by firing inner projectile.

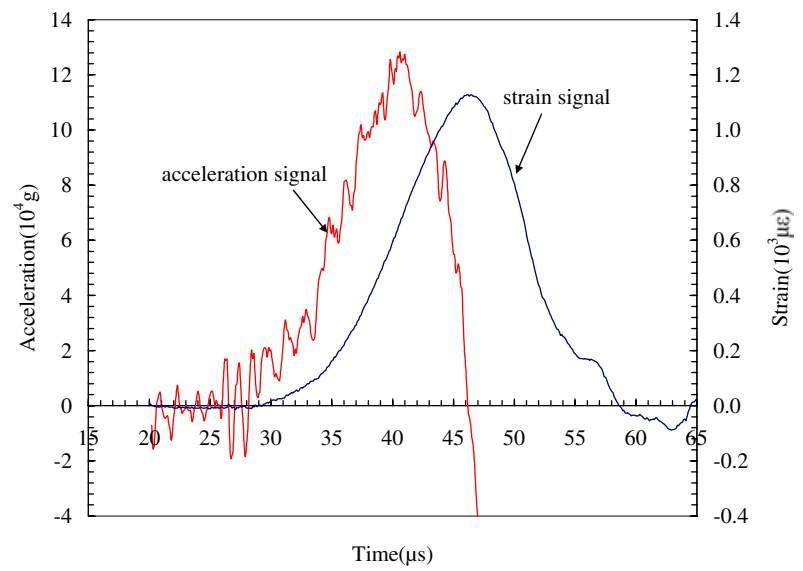

Figure 8. Acceleration and corresponding strain pulses on the bar by firing outer projectile.

two projectiles together without overrun gap are fired and impacted on the end of incident bar at the same time at a fixed velocity of $10 \mathrm{~m} / \mathrm{s}, \varepsilon_{1+2}(t)$ and $g_{1+2}(t)$ are obtained.

The actual operating procedure is as following:

First step, the inner projectile is ahead of outer projectile for $4 \mathrm{~mm}$, and it first impacts on the bar at a fixed velocity of $10 \mathrm{~m} / \mathrm{s}$, Acceleration and corresponding strain pulses obtained as shown in the Fig. 7. The corresponding max acceleration value is about $10.5 \times 10^{4} \mathrm{~g}$.

Second step, the outer projectile is ahead of inner projectile for $4 \mathrm{~mm}$, and it first impacts on the bar at a fixed velocity of $10 \mathrm{~m} / \mathrm{s}$, Acceleration and corresponding strain pulses obtained as shown in the Fig. 8. The corresponding max acceleration value is about $13 \times 10^{4} \mathrm{~g}$.

Third step, the outer projectile is ahead of inner projectile for $0 \mathrm{~mm}$, and they simultaneously impacts on the bar at a fixed velocity of $10 \mathrm{~m} / \mathrm{s}$, Acceleration and corresponding strain pulses obtained as shown in the Fig. 9. The corresponding max acceleration value is about $26 \times 10^{4} \mathrm{~g}$.

In the Fig. 10, the acceleration pulses from first step and second step are added together, that is, an acceleration superimposition pulse, and corresponding strain superimposition are obtained. The corresponding superimposition acceleration value is about $23.5 \times 10^{4} \mathrm{~g}$. 


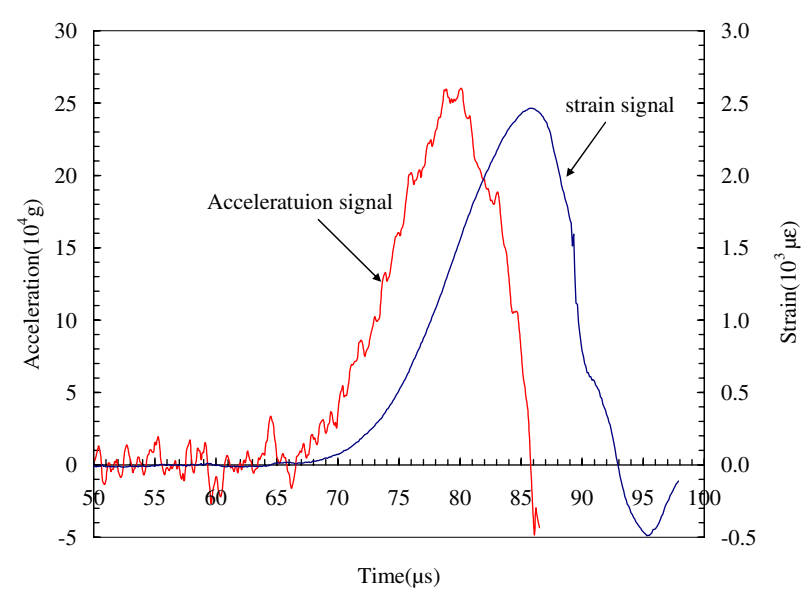

Figure 9. Acceleration and corresponding strain pulses on the bar by firing inner and outer projectiles at the same time.

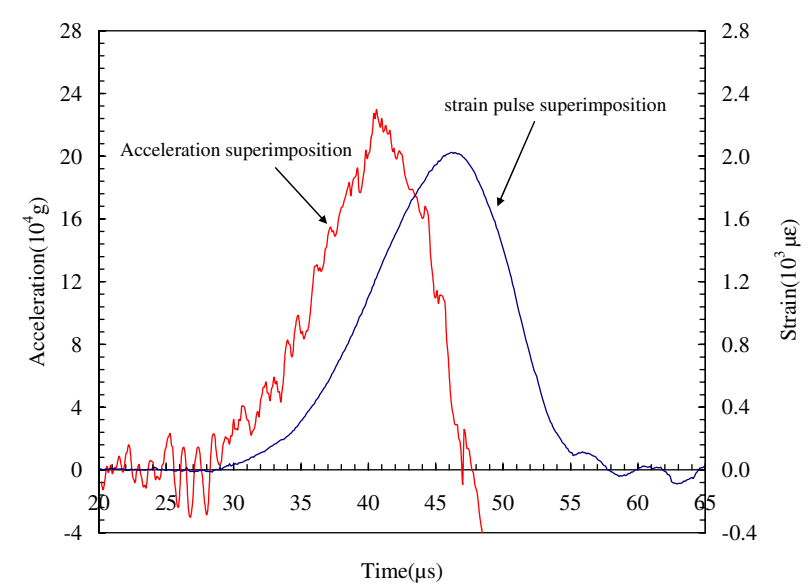

Figure 10. Acceleration and corresponding strain superimposition pulses from two projectile independent impacts on the bars.

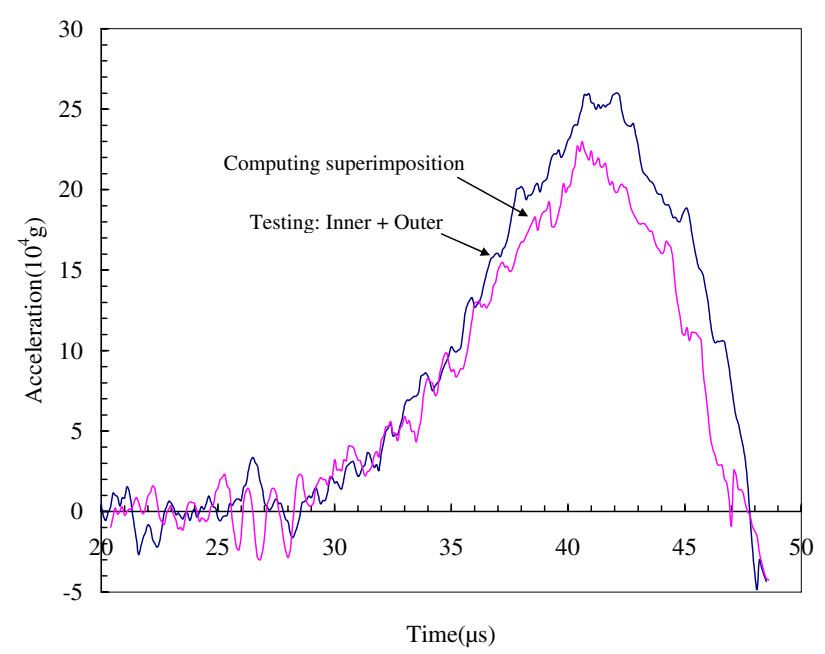

Figure 11. The comparison between testing acceleration pulse and computing superimposition pulses.

this is smaller $2.5 \times 10^{4} \mathrm{~g}$ than direct measurement value of Fig. 9.

In Fig. 11, the comparison between directly testing acceleration pulse and computing superimposition pulses

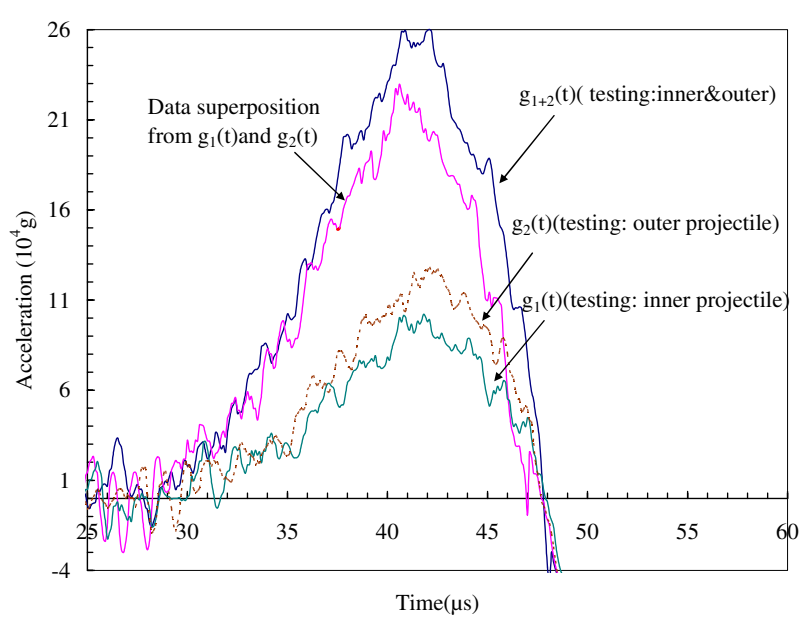

Figure 12. The comparison between testing acceleration pulses of two projectiles and computing superimposition pulses.

is presented, a good agreement of two pulses is obtained except their peak value difference.

In Fig. 12, all acceleration pulses are shown in the same figure, using the calculation method of dynamic linearity of an accelerometer as shown in Fig. 2, and using Eq. (3), the dynamic linearity differences of an accelerometer can be known at any acceleration value point.

\section{Conclusions}

In this work, to measure dynamic linearity of an accelerometer, a single barrel is taken to fire two coaxial cylinders at the any time and at a fixed velocity, the operation and control are easy. Using this method, it can correct a acceleration $\mathrm{g}$ value from order $10^{4} \mathrm{~g}$ to about $20 \times 10^{4} \mathrm{~g}$ in accordance with standard procedure and also can measure dynamic linearity of the accelerometers by choosing different length and cross section area of the projectile. It should be remind that the dispersion on the bar, the front end shape of the coaxial projectiles (outer circular tube and inner cylinder) effect on the stain pulse, the measuring uncertainty etc.. Do not be further presented in the work due to the length limitation of the article.

This work is partly supported from the 111 project of China (B07050).

\section{References}

[1] Robert D. Still, Project Engineer, ENDEVCO. Albuquerque: International Instrumentation Symposium, Instrument Society of America (1983)

[2] T. C. Togami, W. E. Baker, M. J. Forrestal, Appl. Mech 63, 353-356 (1996)

[3] K. Ueda, A. Umeda, in Proceedings of the 9th International Conference on Experimental Mechanics, 1990 , p. 24 
[4] K. Ueda, A. Umeda. Experimental Mechanics 33, 228-233 (1993)

[5] Akira Umeda, Kazunaga Ueda. Method and Apparatus for Measuring Dynamic Response Characteristics of Shock Accelerometer [P]. United States Patent 5000030 (1991)

[6] Yulong Li, Weiguo Guo etc., Explosion and shock waves (in Chinese) 17, 90-96 (1997)

[7] Akira Umeda, Method and Device for Measuring Dynamic Linearity of Acceleration Accelerometer
[P]. United States Patent US2005/0160785 A1, (2005)

[8] CDV draft, Dynamic linearity measurement using an impact acceleration generator, IEC. Committee draft for vote, Project number IEC 60747-14-4 Ed. 1.0, 74-76 (2002)

[9] P. S. Follansbee, and C. Frantz, J. Eng. Mater. Technol 105, 61-67 (1983)

[10] D. J. Frew, M. J. Forrestal, and W. Chen, Experimental 